\title{
Persistent spin dynamics in the pressurized spin-liquid candidate $\mathrm{YbMgGaO}_{4}$
}

\author{
Mayukh Majumder, ${ }^{1}$ Gediminas Simutis, ${ }^{2}$ Ines E. Collings, ${ }^{3}$ Jean-Christophe Orain, ${ }^{2}$ Tusharkanti Dey, ${ }^{1}$ Yuesheng Li, ${ }^{1}$ \\ Philipp Gegenwart, ${ }^{1}$ and Alexander A. Tsirlin ${ }^{1, *}$ \\ ${ }^{1}$ Experimental Physics VI, Center for Electronic Correlations and Magnetism, University of Augsburg, 86159 Augsburg, Germany \\ ${ }^{2}$ Laboratory for Muon Spin Spectroscopy, Paul Scherrer Institut, 5232 Villigen PSI, Switzerland \\ ${ }^{3}$ European Synchrotron Radiation Facility, 71 Avenue des Martyrs, 38000 Grenoble, France
}

(Received 20 February 2019; revised manuscript received 23 April 2020; accepted 28 April 2020; published 19 May 2020)

\begin{abstract}
Single-crystal $\mathrm{x}$-ray diffraction, density functional band structure calculations, and muon spin relaxation $(\mu \mathrm{SR})$ are used to probe the pressure evolution of the triangular spin-liquid candidate $\mathrm{YbMgGaO}_{4}$. The rhombohedral crystal structure is retained up to at least $10 \mathrm{GPa}$ and shows a nearly uniform compression along both the in-plane and out-of-plane directions, whereas local distortions caused by the random distribution of $\mathrm{Mg}^{2+}$ and $\mathrm{Ga}^{3+}$ remain mostly unchanged. The $\mu$ SR data confirm persistent spin dynamics up to $2.6 \mathrm{GPa}$ and down to $250 \mathrm{mK}$ with no change in the muon relaxation rate. Longitudinal-field $\mu$ SR reveals power-law behavior of the spin-spin autocorrelation function, both at ambient pressure and upon compression.
\end{abstract}

DOI: 10.1103/PhysRevResearch.2.023191

\section{INTRODUCTION}

Spin-liquid states in frustrated magnets are nowadays actively studied as hosts for unconventional excitations representing magnetic monopoles [1,2] and other exotic quasiparticles $[3,4]$. One relatively unexplored aspect in this field is the evolution of spin-liquid materials under pressure and the change in spin dynamics caused by tuning magnetic interactions via lattice compression.

Here, we focus on the spin-liquid candidate $\mathrm{YbMgGaO}_{4}$ [5-7] that recently evolved as a unique triangular antiferromagnet with the robust threefold symmetry, persistent spin dynamics, and a broad continuum of (potentially fractionalized) magnetic excitations. The crystal structure of this compound features triangular layers of the pseudospin- $\frac{1}{2} \mathrm{Yb}^{3+}$ ions that are well separated by slabs of nonmagnetic $\mathrm{Mg}^{2+}$ and $\mathrm{Ga}^{3+}$ [Fig. 1(a)]. Thermodynamic measurements [5,6] and muon spin relaxation $(\mu \mathrm{SR})[8]$ suggest the absence of magnetic order down to at least $50 \mathrm{mK}$. Weak spin freezing around $100 \mathrm{mK}$ was indicated by ac-susceptibility data [9], although it involves only a tiny amount of the magnetic entropy [5] and leaves no signatures in either dc susceptibility [10] or $\mu \mathrm{SR}$ [8].

Magnetic excitations of $\mathrm{YbMgGaO}_{4}$ form a broad continuum that can be interpreted in terms of gapless spinons [11-14] or as arising from short-range valence bonds [10,15], the latter suggestion being particularly interesting, as it makes a direct link to Anderson's pioneering work at the outset of the spin-liquid research [16]. Continuum features were also

\footnotetext{
*altsirlin@gmail.com

Published by the American Physical Society under the terms of the Creative Commons Attribution 4.0 International license. Further distribution of this work must maintain attribution to the author(s) and the published article's title, journal citation, and DOI.
}

reported for other $\mathrm{Yb}^{3+}$ triangular antiferromagnets [17-19], but they show a different distribution of the spectral weight and probably have a separate origin. Indeed, $\mathrm{YbMgGaO}_{4}$ is known to be strongly affected by structural randomness that arises from the random distribution of $\mathrm{Mg}^{2+}$ and $\mathrm{Ga}^{3+}$ between the $\mathrm{Yb}^{3+}$ layers and modulates magnetic interactions via random crystal electric fields acting on $\mathrm{Yb}^{3+}[20,21]$. No such randomness occurs in other Yb-based triangular antiferromagnets.

In the following, we explore the effect of hydrostatic pressure on the structure and magnetism of $\mathrm{YbMgGaO}_{4}$, and juxtapose the behavior of this material with the evolution of other spin-liquid candidates upon compression. We show that in $\mathrm{YbMgGaO}_{4}$, spins remain dynamic up to at least $2.6 \mathrm{GPa}$, and quantify associated structural changes for an eventual comparison across different classes of spin-liquid materials.

\section{CRYSTAL STRUCTURE}

\section{A. Average structure}

Room-temperature x-ray diffraction (XRD) data were collected at the ID15B beam line of the European Synchrotron Radiation Facility (ESRF) between ambient pressure and $10 \mathrm{GPa}$. A diamond-anvil cell loaded with $\mathrm{He}$ gas and a small single crystal of $\mathrm{YbMgGaO}_{4}$ from the batch reported in Ref. [6] were used for the experiment.

Three positional parameters ( $z$ coordinates) and three independent atomic displacement parameters were refined for $\mathrm{Mg} / \mathrm{Ga}, \mathrm{O} 1$, and $\mathrm{O} 2$. The position of $\mathrm{Yb}$ was fixed at $(0,0,0)$, with its thermal ellipsoid refined anisotropically to account for local displacements caused by the random distribution of $\mathrm{Mg}$ and $\mathrm{Ga}$. Altogether, nine structural parameters were refined from about 120 symmetry-independent reflections collected at each pressure point (see Supplemental Material) [22]. Details 

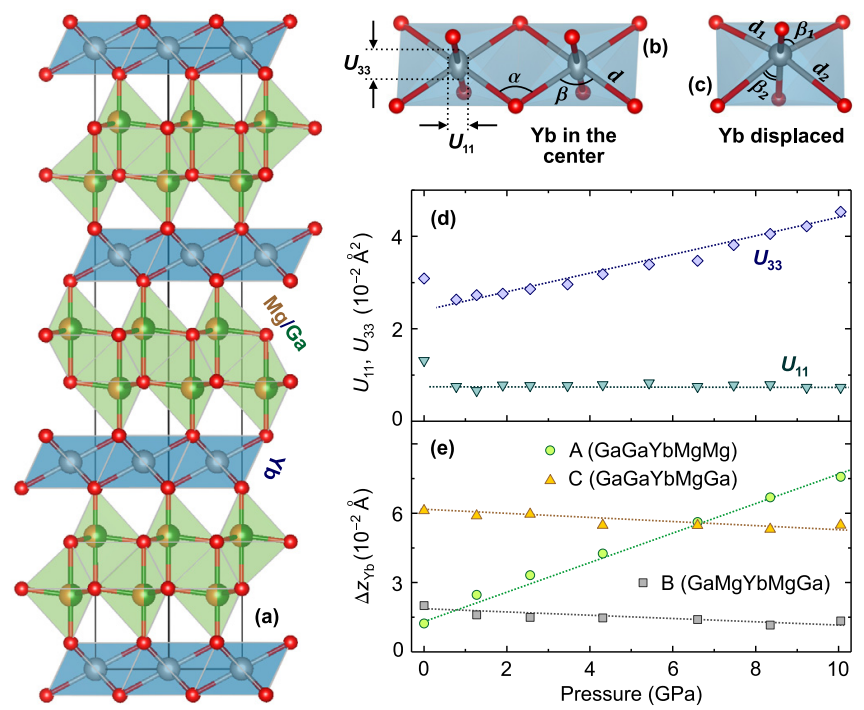

FIG. 1. (a) Crystal structure of $\mathrm{YbMgGaO}_{4}$ with $\mathrm{Mg}^{2+}$ and $\mathrm{Ga}^{3+}$ randomly occupying the position in the trigonal bipyramids. (b) Local structural parameters, the Yb-O distance $(d)$ and $\mathrm{Yb}-\mathrm{O}-$ $\mathrm{Yb}(\alpha)$ or $\mathrm{O}-\mathrm{Yb}-\mathrm{O}(\beta)$ angles with $\alpha=\beta$. (c) Distortion of the $\mathrm{YbO}_{6}$ octahedron caused by an asymmetric distribution of the $\mathrm{Mg}$ and $\mathrm{Ga}$ atoms. (d) Experimental atomic displacement parameters of Yb. (e) Displacements of the Yb atoms obtained for different local configurations shown in Fig. 3.

of the data collection and structure refinement are given in Appendix A.

The $R \overline{3} m$ symmetry of $\mathrm{YbMgGaO}_{4}$ keeps all $\mathrm{Yb}-\mathrm{O}$ distances equal, but allows a trigonal distortion of the $\mathrm{YbO}_{6}$ octahedra with the $\mathrm{O}-\mathrm{Yb}-\mathrm{O}$ angle $\beta$ deviating from $90^{\circ}$. Incidentally, this angle is equal by symmetry to the $\mathrm{Yb}-\mathrm{O}-\mathrm{Yb}$ bridging angle $\alpha$, which is responsible for superexchange interaction [Fig. 1(b)]. Two structural parameters, one distance and one angle, are thus sufficient to characterize both the local geometry of $\mathrm{Yb}^{3+}$ and the geometry of the nearest-neighbor exchange pathway within the average structure.

No changes in the crystal symmetry were observed in our experiment. The pressure evolution of the $a$ and $c$ lattice parameters demonstrates similar compressibility along the different crystallographic directions [Fig. 2(a)]. The fit with the second-order Birch-Murnaghan equation of state,

$$
p(V)=\frac{3 B_{0}}{2}\left[\left(\frac{V_{0}}{V}\right)^{\frac{7}{3}}-\left(\frac{V_{0}}{V}\right)^{\frac{5}{3}}\right],
$$

using the EOSFIT routine [23], returned the bulk modulus of $B_{0}=142(2) \mathrm{GPa}$ and the unit-cell volume of $V_{0}=$ 253.12(7) $\AA^{3}$. A similar fit for individual lattice parameters using $a^{3}$ or $c^{3}$ in place of $V$ yields $B_{0, a}=151(3) \mathrm{GPa}$ and $B_{0, c}=130(2) \mathrm{GPa}$, suggesting a nearly isotropic compression of the structure. $\mathrm{YbMgGaO}_{4}$ is more compressible than $\mathrm{Yb}_{2} \mathrm{Ti}_{2} \mathrm{O}_{7}\left(B_{0}=219 \mathrm{GPa}\right.$ [24] $)$ and other rare-earth pyrochlores that typically feature the bulk moduli in excess of $200 \mathrm{GPa}$ [25].

The $\mathrm{Yb}-\mathrm{O}$ distances shrink by about $0.6 \%$ at $2.6 \mathrm{GPa}$ (the highest pressure of our $\mu$ SR experiment; see Sec. III) and by $1.7 \%$ at $10 \mathrm{GPa}$ [Fig. 2(b)]. The $\alpha=\beta$ angle shows a weak

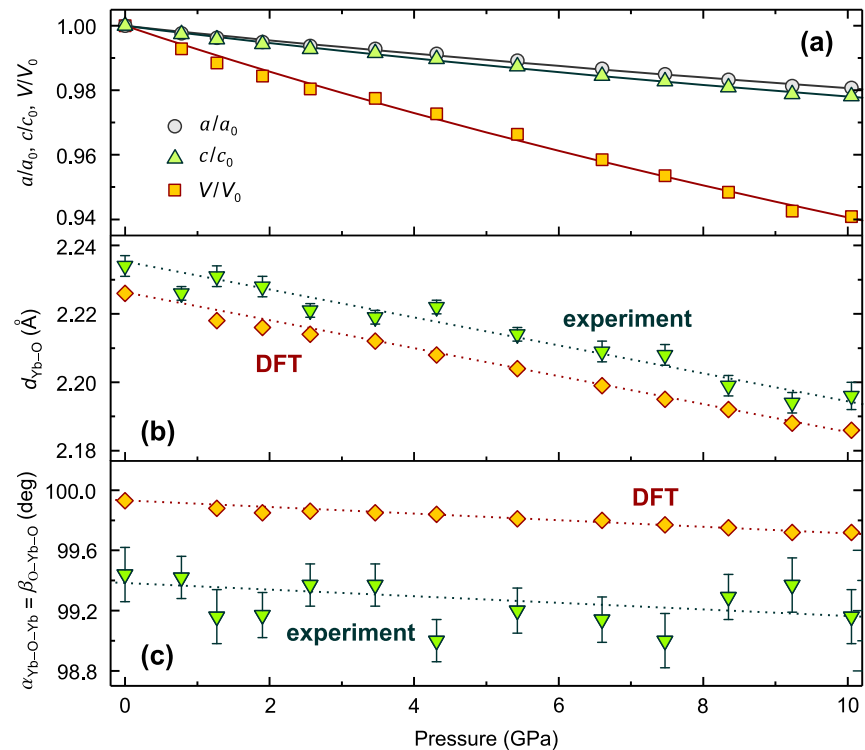

FIG. 2. Pressure-induced changes of the average structure determined using single-crystal XRD: (a) lattice parameters and unit-cell volume; (b) $\mathrm{Yb}-\mathrm{O}$ distance $d$; (c) $\mathrm{Yb}-\mathrm{O}-\mathrm{Yb} / \mathrm{O}-\mathrm{Yb}-\mathrm{O}$ angles $(\alpha=\beta)$. The lines in (a) are fits with the second-order Murnaghan equation of state. (b) and (c) additionally show the geometrical parameters obtained from DFT structure relaxations detailed in Appendix B, and dotted lines are a guide for the eye.

downward trend only, with a larger error bar caused by the lower accuracy in the determination of oxygen position due to the low scattering power of oxygen. In order to confirm this downward trend, we relaxed the experimental structures using density functional theory (DFT) band structure calculations. As DFT cannot treat the $\mathrm{Mg} / \mathrm{Ga}$ disorder explicitly, ordered structural models have to be used, as explained in Appendix B. This approximation leads to a constant offset between the DFT results and experiment. Nevertheless, not only the qualitative trends but also the slope are well reproduced (Fig. 2). We thus confirm that the $\mathrm{Yb}-\mathrm{O}-\mathrm{Yb}$ angle decreases under pressure. Compared to the ambient-pressure value, it changes by $0.07^{\circ}$ at $2.6 \mathrm{GPa}$ and by $0.2^{\circ}$ at $10 \mathrm{GPa}$.

\section{B. Local structure}

Moderate changes of the average crystal structure are accompanied by a strong elongation of the $\mathrm{Yb}$ thermal ellipsoid. The in-plane displacements characterized by $U_{11}$ are not affected by pressure, whereas the out-of-plane displacement component increases by $70 \%$ [Fig. 1(d)]. This out-of-plane displacement has been previously linked to the local distortions of the $\mathrm{YbO}_{6}$ octahedra caused by the random (and, generally, asymmetric) distribution of the differently charged $\mathrm{Mg}^{2+}$ and $\mathrm{Ga}^{3+}$ ions around $\mathrm{Yb}^{3+}$ [20]. At first glance, the increase in $U_{33}$ implies a strong enhancement of the structural randomness under pressure, but the actual situation is more complex.

We analyze the pressure evolution of the local structure by DFT relaxations for several ordered models of $\mathrm{YbMgGaO}_{4}$. First, we consider the models of Ref. [20] that were previously used to interpret crystal-field excitations of $\mathrm{Yb}^{3+}$, and evaluate 

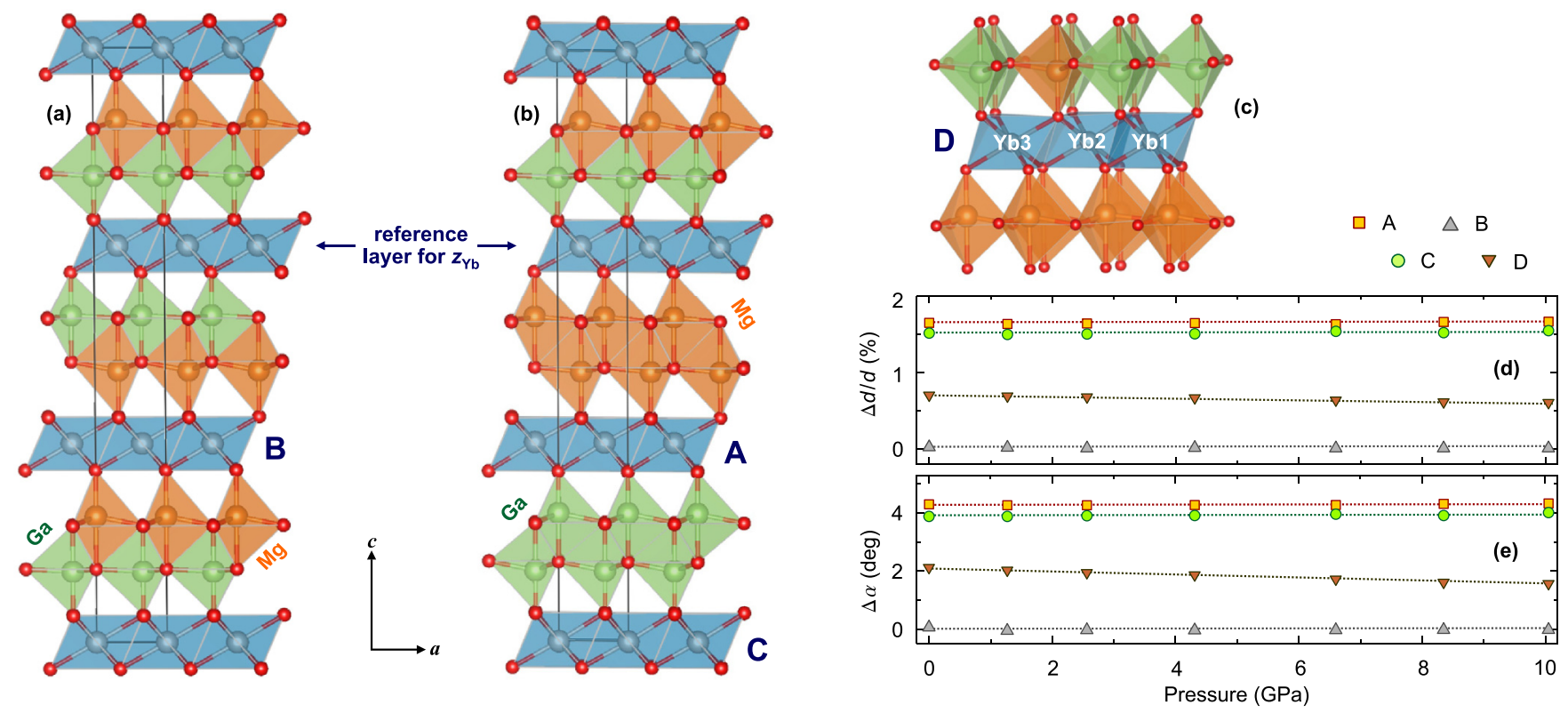

FIG. 3. Ordered models for evaluating pressure evolution of the local structure. (a),(b) Layer-by-layer ordering of $\mathrm{Mg}^{2+}$ and $\mathrm{Ga}^{3+}$ leading to the $\mathrm{Yb}$ layers $\mathrm{A}, \mathrm{B}$, and $\mathrm{C}$ with the same $\mathrm{YbO}_{6}$ octahedra throughout each layer. (c) A more complex model with layer $\mathrm{D}$ containing nonequivalent $\mathrm{YbO}_{6}$ octahedra. (d) Relative deformations of the octahedra obtained as $\left(d_{1}-d_{2}\right) /\left(d_{1}+d_{2}\right)$ for layers $\mathrm{A}$, B, and $\mathrm{C}$ and as a difference between the average $d$ for the $\mathrm{Yb} 1$ and $\mathrm{Yb} 2$ octahedra in layer $\mathrm{D}$. (e) Angular deformation obtained as $\Delta \alpha=\Delta \beta=\beta_{2}-\beta_{1}$ for layers $\mathrm{A}, \mathrm{B}$, and $\mathrm{C}$ and as the difference in the $\mathrm{Yb} 1-\mathrm{Yb} 2$ and $\mathrm{Yb} 2-\mathrm{Yb} 3$ superexchange angles for layer D.

$\Delta z_{\mathrm{Yb}}$ as the displacements of the $\mathrm{Yb}$ atoms relative to each other. These displacements indicate the spread of the $\mathrm{Yb}$ electron density, which is gauged by the $U_{33}$ parameter of the average structure.

Three scenarios visualized in Figs. 3(a) and 3(b) are considered, with the $\mathrm{Yb}^{3+}$ ions sandwiched between the following: (i) two $\mathrm{Ga}^{3+}$ ions on one side and two $\mathrm{Mg}^{2+}$ ions on the other side (octahedra A), (ii) one $\mathrm{Ga}^{3+}$ and one $\mathrm{Mg}^{2+}$ ion on each side (octahedra B), and (iii) two $\mathrm{Ga}^{3+}$ ions on one side and a combination of $\mathrm{Ga}^{3+}$ and $\mathrm{Mg}^{2+}$ on the other side (octahedra $\mathrm{C}$ ). Pressure has a strong effect on the $\mathrm{Yb}$ position in the octahedra $\mathrm{A}$ and nearly no effect on the $\mathrm{Yb}$ atoms in B and C [Fig. 1(e)]. Surprisingly, this effect is a shift of the whole octahedron A along the $c$ direction without any change in the octahedron itself. Using the geometrical parameters shown in Fig. 1(c), we determine that for octahedra A, the deformation expressed by $\left(d_{2}-d_{1}\right)$ changes from $1.65 \%$ at $0 \mathrm{GPa}$ to $1.66 \%$ at $10 \mathrm{GPa}$, whereas $\left(\beta_{2}-\beta_{1}\right)$ evolves from $4.29^{\circ}$ to $4.32^{\circ}$ [Figs. 3(d) and 3(e)]. The changes in octahedra $\mathrm{B}$ and $\mathrm{C}$ are equally small. Since $\alpha=\beta$ in our material, the distribution of the $\mathrm{Yb}-\mathrm{O}-\mathrm{Yb}$ angles also does not change with pressure. Therefore, neither local randomness at each $\mathrm{Yb}$ site nor randomness of the exchange couplings should be affected by pressure.

We also consider a different scenario where two adjacent $\mathrm{Yb}$ atoms within the same layer have a different local environment. In layer D [Fig. 3(c)], Yb1 is surrounded by $3 \mathrm{Ga}$ atoms on one side and $3 \mathrm{Mg}$ atoms on the other side. In contrast, $\mathrm{Yb} 2$ and $\mathrm{Yb} 3$ are surrounded by $2 \mathrm{Ga}$ and $4 \mathrm{Mg}$ atoms each. These dissimilar local environments lead to different $\alpha_{1}$ and $\alpha_{2}$ for the $\mathrm{Yb} 1-\mathrm{Yb} 2$ and $\mathrm{Yb} 2-\mathrm{Yb} 3$ superexchange pathways and reflect the effect of structural randomness on nearestneighbor magnetic interactions in $\mathrm{YbMgGaO}_{4}$. However, the difference between these two pathways appears to be only weakly pressure-dependent and decreases upon compression [Fig. 3(e)].

Our results suggest that the visible enhancement of $U_{33}$ under pressure is not related to the increased distortions around the $\mathrm{Yb}^{3+}$ ions. It rather indicates a change in the overall position of octahedra $\mathrm{A}$ that may be explained by the accumulation of different charges above $\left(\mathrm{Ga}^{3+}\right)$ and below $\left(\mathrm{Mg}^{2+}\right)$ the $\mathrm{Yb}$ layer. But the key result at this juncture is that such a change reflects a rearrangement within the $\mathrm{Mg} / \mathrm{Ga}$ slabs and does not affect the structure of the magnetic layer itself. The $\mathrm{YbO}_{6}$ octahedra undergo a uniform compression and simply keep the deformation that they had at ambient pressure. The randomness effect on the exchange couplings should be largely unchanged or even decrease under pressure.

\section{SPIN DYNAMICS}

The pressure evolution of spin dynamics is probed by muon spin relaxation $(\mu \mathrm{SR})$. The experiments were performed at the GPD and Dolly spectrometers at the Paul Scherrer Institute (PSI) at ambient pressure and at the GPD spectrometer under hydrostatic pressure [26] down to $250 \mathrm{mK}$ on a polycrystalline sample of $\mathrm{YbMgGaO}_{4}$. The data were collected in zero field (ZF) and in the longitudinal-field (LF) mode, where the magnetic field was applied parallel to the spin of the implanted muons.

\section{A. ZF $\mu$ SR}

$\mathrm{ZF} \mu \mathrm{SR}$ time spectra measured at ambient pressure and at $2.6 \mathrm{GPa}$, the highest pressure of our experiment, are compared 

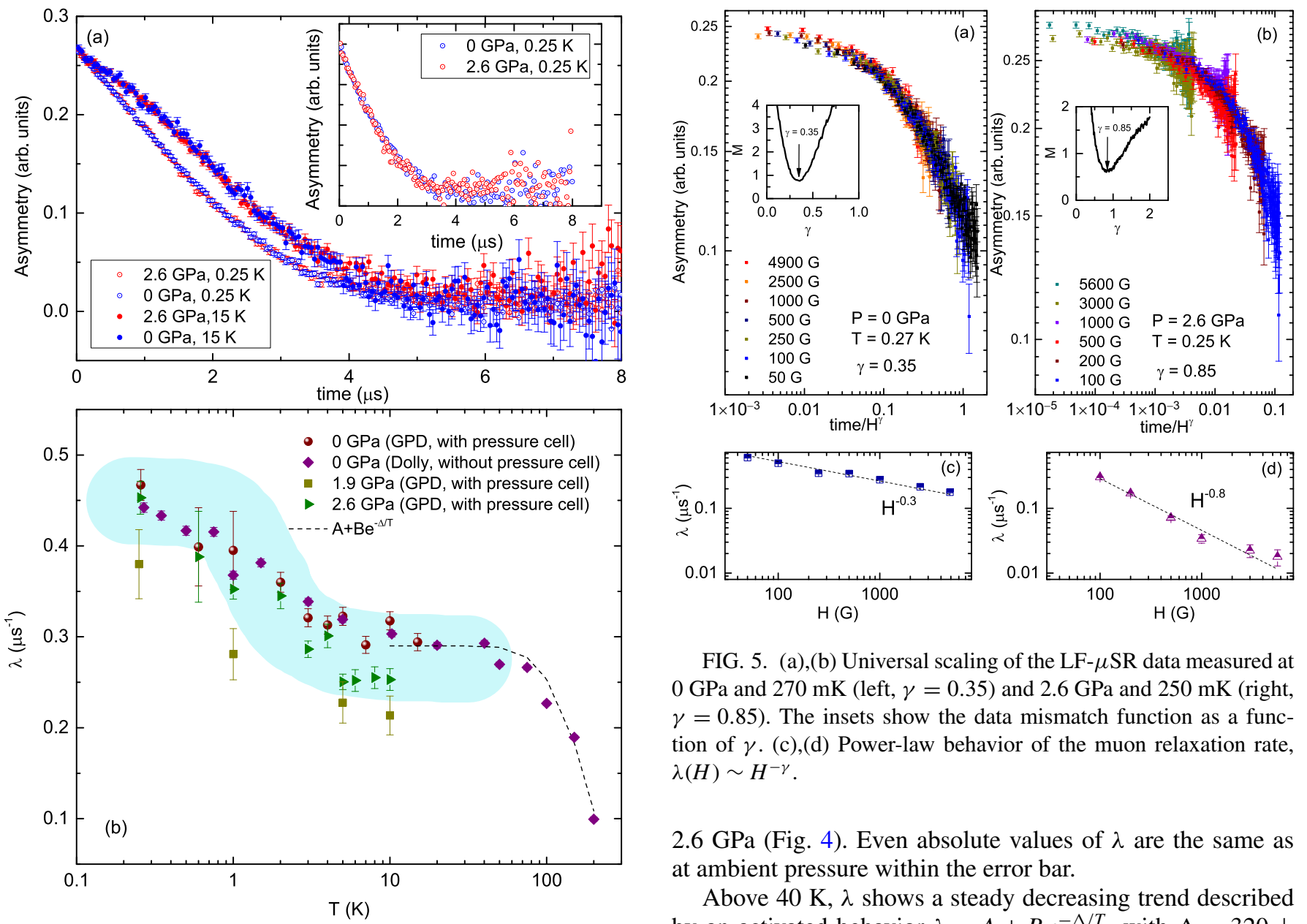

FIG. 5. (a),(b) Universal scaling of the LF- $\mu$ SR data measured at $0 \mathrm{GPa}$ and $270 \mathrm{mK}$ (left, $\gamma=0.35$ ) and $2.6 \mathrm{GPa}$ and $250 \mathrm{mK}$ (right, $\gamma=0.85$ ). The insets show the data mismatch function as a function of $\gamma$. (c),(d) Power-law behavior of the muon relaxation rate, $\lambda(H) \sim H^{-\gamma}$.

2.6 GPa (Fig. 4). Even absolute values of $\lambda$ are the same as at ambient pressure within the error bar.

Above $40 \mathrm{~K}, \lambda$ shows a steady decreasing trend described by an activated behavior $\lambda=A+B e^{-\Delta / T}$, with $\Delta=320 \pm$ $20 \mathrm{~K}$, which is reminiscent of the lowest crystal-field excitation energy of about $450 \mathrm{~K}$ [20]. This observation suggests that at high temperatures, the relaxation is governed by an Orbach process [27] involving the excited crystal-field doublets of $\mathrm{Yb}^{3+}$.

\section{B. LF $\mu$ SR}

in Fig. 4. The similar behavior of the $\mu$ SR time spectra indicates no change of the magnetic ground state, and the absence of oscillations excludes pressure-induced magnetic ordering in $\mathrm{YbMgGaO}_{4}$ up to at least $2.6 \mathrm{GPa}$. To estimate the temperature dependence of the relaxation rate $\lambda$, we fitted the ZF- $\mu$ SR time spectra by

$$
A(t)=f_{\mathrm{PC}} A_{\mathrm{PC}}+\left(1-f_{\mathrm{PC}}\right) e^{-\lambda t},
$$

where $A_{\mathrm{PC}}$ is the pressure-cell contribution described in Appendix $\mathrm{C}$. The fraction of signal coming from the pressure cell was $f_{\mathrm{PC}}=0.7$ at 0 and $2.6 \mathrm{GPa}$, and $f_{\mathrm{PC}}=0.5$ at $1.9 \mathrm{GPa}$, where a different cell was used in an attempt to reduce the background.

The zero-field muon relaxation rate $\lambda$ is temperatureindependent between 4 and $40 \mathrm{~K}$ (Fig. 4). Its increase below $4 \mathrm{~K}$ indicates the onset of spin-spin correlations that fully develop around $0.8 \mathrm{~K}$, where $\lambda$ flattens out and remains temperature-independent upon further cooling. This temperature evolution is essentially similar to the ambient-pressure $\mu \mathrm{SR}$ data reported in Ref. [8] and remains unchanged at

LF measurements complement the data obtained in the zero field. Even a longitudinal field, which is more than 10 times higher than the local static field estimated from the low-temperature value of $\lambda$, does not decouple the muon relaxation, suggesting that the spins are dynamic in nature as has been seen at ambient pressure [8].

The LF data measured in different fields follow universal scaling initially proposed by Keren et al. [28] for systems with glassy dynamics. This scaling manifests itself in the powerlaw behavior of the muon relaxation rate $\lambda(H)$ extracted by fitting individual LF curves with a stretched exponential $e^{-[\lambda(H) t]^{\beta}}$ supplied with the time-independent and fieldindependent background, which originates from the sample holder and pressure cell. The ensuing values of $\lambda$ follow $\lambda(H) \sim H^{-\gamma}$ with $\gamma \simeq 0.3$ at $0 \mathrm{GPa}$ and 0.8 at $2.6 \mathrm{GPa}$ (Fig. 5).

This behavior is confirmed by the scaling of muon asymmetry plotted against $t / H^{\gamma}$. To this end, the data points from all fields up to $t=6 \mu \mathrm{s}$ [29] are arranged with increasing $t / H^{\gamma}$ for every value of $\gamma$. An empirical data mismatch function is calculated by taking the difference between the 
neighboring points and weighing them by the corresponding error bars. This mismatch function is defined as

$$
M=\frac{1}{N} \sum_{i}^{N} \frac{\left(A_{i}-A_{i+1}\right)^{2}}{\left(\delta_{i}-\delta_{i+1}\right)^{2}},
$$

where $N$ is the number of data points, and $A_{i}$ and $\delta_{i}$ correspond to the asymmetry and error bar of the $i$ th data point, respectively. The lowest value of the mismatch function is obtained at $\gamma=0.35(0 \mathrm{GPa})$ and $\gamma=0.85(2.6 \mathrm{GPa})$ that produce the universal scaling over at least three orders of magnitude in $t / H^{\gamma}$ and show excellent agreement with the $\gamma$ values from the analysis of $\lambda(H)$.

The scaling may not hold in the whole time and field range-e.g., at very short times comparable to the width of the muon pulse [28] or in very high fields that affect spin dynamics — but the scaling over three orders of magnitude (Fig. 5) is congruent with all earlier observations [30,31] and serves as a robust evidence of the power-law behavior $q(t) \sim t^{\gamma-1}$ of the dynamic spin-spin autocorrelation function $q(t)=\left\langle S_{i}(t) \cdot S_{i}(0)\right\rangle$. It indicates collective dynamics, as, for example, in spin-glass systems where $\gamma$ increases toward 1.0 upon approaching the freezing temperature from above [28].

\section{DISCUSSION AND SUMMARY}

$\mathrm{YbMgGaO}_{4}$ is remarkably insensitive to pressure and thus different from other spin-liquid candidates. For example, herbertsmithite $[32,33]$ becomes magnetically ordered at $2.5 \mathrm{GPa}$ [34], whereas stoichiometric $\mathrm{Yb}_{2} \mathrm{Ti}_{2} \mathrm{O}_{7}$ [35] shows pressureinduced magnetic order already at $0.1 \mathrm{GPa}$ [36]. These materials have different compressibilities, with the bulk modulus of $B_{0}=70 \mathrm{GPa}$ in herbertsmithite [34] and $B_{0}=219 \mathrm{GPa}$ in $\mathrm{Yb}_{2} \mathrm{Ti}_{2} \mathrm{O}_{7}$ [24]. $\mathrm{YbMgGaO}_{4}$ shows an intermediate value of $142 \mathrm{GPa}$ and is certainly comparable to other spin-liquid candidates as far as elastic properties are concerned. Its structural parameters are affected by pressure. The persistence of spin dynamics will then indicate that structural changes influence exchange couplings only weakly, whereas the material lies sufficiently far away from the phase boundary between the spin-liquid and magnetically ordered states. Alternatively, spin dynamics may not be caused by frustration that controls this phase boundary, and originate from structural randomness, which is not affected by pressure.

Triangular antiferromagnets develop $120^{\circ}$ order or stripe order, depending on the extent of exchange anisotropy [37] and on the ratio $J_{2} / J_{1}$ of the second-neighbor to nearestneighbor exchange coupling [38,39]. The formation of a spin liquid is mostly controlled by $J_{2} / J_{1}$, with the complete suppression of magnetic order between $J_{2} / J_{1} \simeq 0.07$ and 0.15 $[7,38,39]$. Experimental estimates of this parameter vary in a broad range between 0.0 and 0.26 [7] and would place $\mathrm{YbMgGaO}_{4}$ in any of the regions on the phase diagram: $120^{\circ}$ order [6], stripe order [21,40], or the boundary between the latter and the spin-liquid phase [41].

External pressure of 2.6 GPa leads to a shortening of the $\mathrm{Yb}-\mathrm{O}$ distance $d$ by $0.013 \AA$ and a reduction in the $\mathrm{Yb}-\mathrm{O}-\mathrm{Yb}$ angle $\alpha$ by $0.07^{\circ}$ (Fig. 2). Using the superexchange theory of Ref. [42], we estimate that the change in the angle increases $J_{1}$ by $6 \%$, whereas the shortening of the $\mathrm{Yb}-\mathrm{O}$ distance increases $J_{1}$ by another $5 \%$ assuming exponential dependence of Slater-Koster parameters on the interatomic distance. Overall, we expect that $J_{1}$ increases by $11 \%$, which is similar in magnitude to pressure-induced changes in $\mathrm{Cu}$-based quantum magnets $[43,44]$. No significant changes are expected for $J_{2}$ because long-range couplings are less sensitive to the structural geometry. In $\mathrm{YbMgGaO}_{4}$, the $\mathrm{Yb}-\mathrm{O}-\mathrm{O}$ angles decrease by as little as $0.11^{\circ}$ upon compression to $2.6 \mathrm{GPa}$. In contrast, in a Cu-based quantum magnet, even the $2^{\circ}$ change of the respective angle reduces the coupling by only $20 \%$ [45].

We conclude that $J_{1}$ increases, $J_{2}$ is roughly unchanged, and the $J_{2} / J_{1}$ ratio of $\mathrm{YbMgGaO}_{4}$ should be reduced under pressure. Assuming the parametrization of Ref. [41] with $J_{2} / J_{1}=0.18$, it would imply that pressure pushes the material into the spin-liquid region, in agreement with the persistence of spin dynamics observed experimentally. Alternatively, and perhaps even more likely, the lack of significant changes under pressure may indicate that the spin dynamics is triggered by structural randomness and thus unaffected by pressure.

The only change we observe is the increase in the $\gamma$ parameter that describes scaling behavior in longitudinal fields. For a system with glassy dynamics, the increase in $\gamma$ would reflect an evolution toward a frozen state [28] that was indeed proposed for $\mathrm{YbMgGaO}_{4}$ below $100 \mathrm{mK}$ (at ambient pressure) based on the ac-susceptibility data [9]. However, neither dc susceptibility [10] nor $\mu$ SR [8] support bulk spin freezing at ambient pressure. The scaling behavior may, in fact, be unrelated to glassy dynamics, and indeed it was also reported for a variety of systems with critical fluctuations influenced by structural disorder $[30,31]$. On the other hand, the very presence of this scaling serves as an additional argument for collective spin dynamics caused by the structural randomness, and the importance of this randomness for the spin-liquid behavior of $\mathrm{YbMgGaO}_{4}$.

Altogether, hydrostatic pressure leads to a uniform compression of the $\mathrm{YbMgGaO}_{4}$ structure with the reduction in the $\mathrm{Yb}-\mathrm{O}$ distances and $\mathrm{Yb}-\mathrm{O}-\mathrm{Yb}$ angles, whereas local distortions of the $\mathrm{YbO}_{6}$ octahedra and consequent randomness effects are nearly unchanged. Spin dynamics is not affected by pressure and appears to be collective yet influenced by the structural randomness. This puts $\mathrm{YbMgGaO}_{4}$ into the group of materials where randomness effects can be integral to the spin-liquid formation, but collective spin dynamics is nevertheless observed.

\section{ACKNOWLEDGMENTS}

We acknowledge ESRF for provision of beam time at ID15B. I.E.C. thanks J. Jacobs for the He gas load. Part of this work is based on experiments performed at the Swiss Muon Source $\mathrm{S} \mu \mathrm{S}$, Paul Scherrer Institute, Villigen, Switzerland. The Swiss National Science Foundation has supported the work of G.S. (Grant No. 200021-175935 and Mobility Grant No. P2EZP2-178604). The work in Augsburg was supported by the German Research Foundation (DFG) via Project No. 107745057 (TRR80) and by the Federal Ministry for Education and Research through the Sofja Kovalevkaya Award of Alexander von Humboldt Foundation (A.A.T.). 
TABLE I. Details of the single-crystal XRD data collection and structure refinement at $10 \mathrm{GPa}$.

\begin{tabular}{lc}
\hline \hline$T(\mathrm{~K})$ & 298 \\
$a(\AA)$ & $3.34272(13)$ \\
$c(\AA)$ & $24.6033(7)$ \\
Wavelength $(\AA)$ & 0.41114 \\
$h_{\min }, h_{\max }$ & $-4 \leqslant h \leqslant 3$ \\
$k_{\min }, k_{\max }$ & $-5 \leqslant k \leqslant 5$ \\
$l_{\min }, l_{\max }$ & $-39 \leqslant l \leqslant 38$ \\
No. of reflections & 126 \\
No. of refinable parameters & 9 \\
$R_{\text {int }}$ & 0.0249 \\
$R_{I}$ & 0.0310 \\
\hline \hline
\end{tabular}

\section{APPENDIX A: X-RAY DIFFRACTION}

Membrane-driven LeToullec-type diamond-anvil cells (DACs) were used, equipped with Boehler-Almax anvils. Stainless steel was used as the gasket material and helium was loaded as the pressure-transmitting medium. Diffraction patterns were collected with a Mar555 flat-panel detector using steps of $0.5^{\circ}$ oscillations over a total $\omega$ scan range of $76^{\circ}$ about the vertical axis. The pressures were measured using the ruby fluorescence method. Lattice parameter determination and integration of the reflection intensities were performed using the CRYSALISPRO software [46]. Structures were refined using SHELXL [47] within the SHELXLE [48] graphical interface.

Details of the data collection and structure refinement at $10 \mathrm{GPa}$ are listed in Table I. The refinements at other pressures were similar and can be found in the cif file provided as Supplemental Material [22]. We note that the ambient-pressure experiment was performed before loading the cell with the $\mathrm{He}$ gas. This may be the reason for the abrupt change in the $\mathrm{Yb}$ displacement parameters $U_{11}$ and $U_{33}$ between 0 and $0.78 \mathrm{GPa}$ [Fig. 1(d)].

\section{APPENDIX B: COMPUTATIONAL RESULTS}

\section{General methodology}

Nonmagnetic DFT calculations were performed in the FPLO [49] and VASP [50] codes. Atomic positions were optimized until the energy minimum was reached, and residual forces dropped below $0.005 \mathrm{eV} / \AA$. The typical $k$ mesh included 64 points within the first Brillouin zone. Experimental lattice parameters were used at each pressure, and only atomic positions were relaxed.

The results of structure optimization may be affected by the computational methodology. In Table II, we analyze the role of three factors: (i) band structure code, (ii) exchangecorrelation potential, and (iii) treatment of the $\mathrm{Yb} 4 f$ shell. The calculations are performed for the simplest $R 3 m$ ordered model of $\mathrm{YbMgGaO}_{4}$ described in Appendix B 2 .

The choice of the band structure code affects the results of the relaxation. VASP calculations predict the too-long $\mathrm{Yb}-\mathrm{O}$ distances and, consequently, the too-low angles. On the other hand, the relaxations within FPLO produce, irrespective of the methodology, the average distance $\left(d_{1}+d_{2}\right) / 2$ and the average angle $\left(\beta_{1}+\beta_{2}\right) / 2$ within, respectively, $0.01 \AA$ and $0.5^{\circ}$ from the experimental values. The larger deviations of the VASP results are probably related to the lower accuracy of the default pseudopotential for the $\mathrm{Yb}$ atoms, whereas FPLO does not rely on pseudopotentials and introduces no approximations to the crystal potential.

All choices mentioned here, including the VASP calculations, produce qualitatively similar results as a function of pressure. The trends obtained from DFT are thus robust. The results shown in the manuscript are obtained with FPLO, GGA functional, and $\mathrm{Yb} 4 f$ states placed into the core, as this choice improved the convergence for larger unit cells.

\section{Ordered structural models}

As DFT cannot treat mixed sites, ordered structural models are used for calculations. We adopted three types of such ordered models:

(1) The $R 3 m$ model obtained from the parent $R \overline{3} m$ structure by splitting the mixed $\mathrm{Mg} / \mathrm{Ga}$ site into two: one fully occupied by $\mathrm{Ga}$ and the other one fully occupied by $\mathrm{Mg}$. In this case, each $\mathrm{Yb}$ layer is sandwiched between the $\mathrm{Mg}$ and Ga layers, leading to the off-center $\mathrm{Yb}$ displacement, two nonequivalent Yb-O distances $d_{1} \neq d_{2}$, and two $\mathrm{O}-\mathrm{Yb}-\mathrm{O}$ angles $\beta_{1} \neq \beta_{2}$. The $R 3 m$ structure was used in Table II for testing the effect of band structure code, basis set, and exchange-correlation potential.

(2) $P 3 m$ models that preserve threefold symmetry and feature layer-by-layer ordering of $\mathrm{Mg}$ and $\mathrm{Ga}$. We chose two of such ordered structures (Fig. 3) that correspond to the third and fourth structures from Fig. S5 of Ref. [20].

In the structure from Fig. 3(a), all $\mathrm{Yb}$ atoms develop a nearly symmetric local environment. This structure was used

TABLE II. Comparison of the relaxed geometrical parameters for the ambient-pressure crystal structure in the primitive cell with the $R 3 m$ symmetry. Different band structure codes (FPLO vs VASP), exchange-correlation potentials (local density approximation (LDA) [51] vs generalized gradient approximation (GGA) [52]), and approaches to the treatment of the Yb $4 f$ shell (valence vs core) have been used.

\begin{tabular}{lccccrr}
\hline \hline Code & FPLO & FPLO & FPLO & FPLO & VASP & Experiment \\
\hline$V_{\mathrm{xc}}$ & LDA & LDA & GGA & GGA & GGA \\
$\mathrm{Yb} 4 f$ & valence & core & valence & core & core \\
$d_{1} / d_{2}(\AA)$ & $2.229 / 2.248$ & $2.214 / 2.233$ & $2.231 / 2.257$ & $2.215 / 2.243$ & $2.258 / 2.270$ \\
$\beta_{1} / \beta_{2}(\mathrm{deg})$ & $98.62 / 99.73$ & $99.52 / 100.68$ & $98.09 / 99.60$ & $98.88 / 100.62$ & $97.31 / 98.02$ & $99.44(13)$ \\
\hline \hline
\end{tabular}


to evaluate the pressure dependence of the $\mathrm{Yb}-\mathrm{O}$ distances and $\mathrm{Yb}-\mathrm{O}-\mathrm{Yb}$ angles shown in Fig. 2. In this case, the octahedra are still deformed, but $d_{2}-d_{1}<0.005 \AA$ and $\beta_{2}-\beta_{1}<0.2^{\circ}$ indicate a negligibly small deformation. The "symmetric" structure (left panel of Fig. 3) is also used to obtain the displacements $\left(\Delta z_{\mathrm{Yb}}\right)$ of $\mathrm{Yb}$ within octahedra B $(\mathrm{GaMgYb}-$ $\mathrm{MgGa})$.

The structure from Fig. 3(b) covers the opposite scenario of the $\mathrm{Yb}$ atoms with a highly asymmetric local environment. It contains octahedra $\mathrm{A}(\mathrm{GaGaYbMgMg})$ and $\mathrm{C}(\mathrm{GaGaYb}-$ $\mathrm{MgGa}$ ) showing the largest values of $d_{2}-d_{1}$ and $\beta_{2}-\beta_{1}$. As explained in the main text, the thermal displacement parameter of the average structure, $U_{33}$, does not reflect deformations of individual octahedra and instead shows relative displacements of the $\mathrm{Yb}$ atoms with respect to each other. In the ideal structure, the $\mathrm{Yb}$ atoms should be at $z=0, \frac{1}{3}, \frac{2}{3}$. To assess the effect of pressure on $U_{33}$, we compare the positions of the $\mathrm{Yb}$ atoms within the octahedra $\mathrm{A}$ and $\mathrm{C}$ to the remaining $\mathrm{Yb}$ atom at $z \simeq \frac{2}{3}$ that shows a rather symmetric local environment and can be used as reference. The values of $\Delta z_{\mathrm{Yb}}$ are obtained as $\left|z_{A, C}-z_{\text {ref }}-\frac{1}{3}\right|$, where $z_{\text {ref }}$ is the $z$ coordinate of the reference $\mathrm{Yb}$ atom.

(3) The $P 3 m$ model with the fourfold $2 \mathbf{a} \times 2 \mathbf{b} \times \mathbf{c}$ supercell, where $\mathbf{a}, \mathbf{b}$, and $\mathbf{c}$ are lattice vectors of the parent $R \overline{3} m$ structure. This supercell allows one to construct configurations where adjacent $\mathrm{Yb}$ atoms have a different local environment. We focus on the fragment shown in Fig. 3(c), where $\mathrm{Yb} 1-\mathrm{Yb} 2$ and $\mathrm{Yb} 2-\mathrm{Yb} 3$ represent two nonequivalent superexchange pathways caused by the uneven distribution of $\mathrm{Mg}$ and $\mathrm{Ga}$ around the $\mathrm{Yb}$ layer.

\section{APPENDIX C: $\mu$ SR EXPERIMENT}

$\mu \mathrm{SR}$ measurements were performed in double-walled MP35 pressure cells with Daphne oil 7373 as the pressuretransmitting medium. The pressure value was determined by measuring the superconducting transition of a small piece of
TABLE III. Details of the $\mu$ SR experiments.

\begin{tabular}{lcccc}
\hline \hline$P(\mathrm{GPa})$ & Spectrometer & Pressure cell & $T(\mathrm{~K})$ & Mode \\
\hline 0 & Dolly & none & $0.26-200$ & $\mathrm{ZF} / \mathrm{LF}$ \\
0 & GPD & MP35N + MP35N & $0.25-10$ & ZF \\
1.9 & GPD & MP35N + CuBe & $0.25-10$ & ZF \\
2.6 & GPD & MP35N + MP35N & $0.25-10$ & ZF/LF \\
\hline \hline
\end{tabular}

indium positioned next to the sample inside the pressure cell. Further experimental details are summarized in Table III.

Two different pressure cells were used. The 0 and 2.6 GPa data were collected in the double-walled MP35N+MP35Ntype cell [26], whereas the $1.9 \mathrm{GPa}$ data were collected separately in the low-background double-walled MP35N+CuBe-type cell [53]. For both cells, we employed Eq. (2) and expressed the pressure-cell contribution as the Kubo-Toyabe depolarization function multiplied by an exponential damping,

$$
A_{\mathrm{PC}}(t)=\left[\frac{1}{3}+\frac{2}{3}\left(1-\sigma_{\mathrm{PC}}^{2} t^{2}\right) e^{-\sigma_{\mathrm{PC}}^{2} t^{2} / 2}\right] \exp \left(-\lambda \lambda_{\mathrm{PC}} t\right) .
$$

In the case of the MP35N+MP35N-type cell, $\sigma_{\mathrm{PC}}=$ $0.29 \mu \mathrm{s}^{-1}$ was temperature independent, whereas $\lambda_{\mathrm{PC}}$ remained constant down to $1 \mathrm{~K}$ and increased at lower temperatures, similar to Ref. [26]. The fraction of the signal coming from the pressure cell was $f_{\mathrm{PC}}=0.7$. In the case of the MP35N+CuBe-type cell, both $\sigma_{\mathrm{PC}}=0.32 \mu \mathrm{s}^{-1}$ and $\lambda_{\mathrm{PC}}=0.025 \mu \mathrm{s}^{-1}$ are temperature independent, in agreement with those of Ref. [53], and $f_{\mathrm{PC}}=0.5$. The usage of different cells may be the reason for a slight offset between the $1.9 \mathrm{GPa}$ data and 2.6 GPa data in Fig. 4.

In longitudinal fields above $100 \mathrm{G}$, the contribution of the pressure cell should be mostly decoupled, and the data include only a constant, field-independent background. Figure 6 shows the LF data fitted by the stretched exponentials to obtain $\lambda(H)$ shown in Fig. 5.
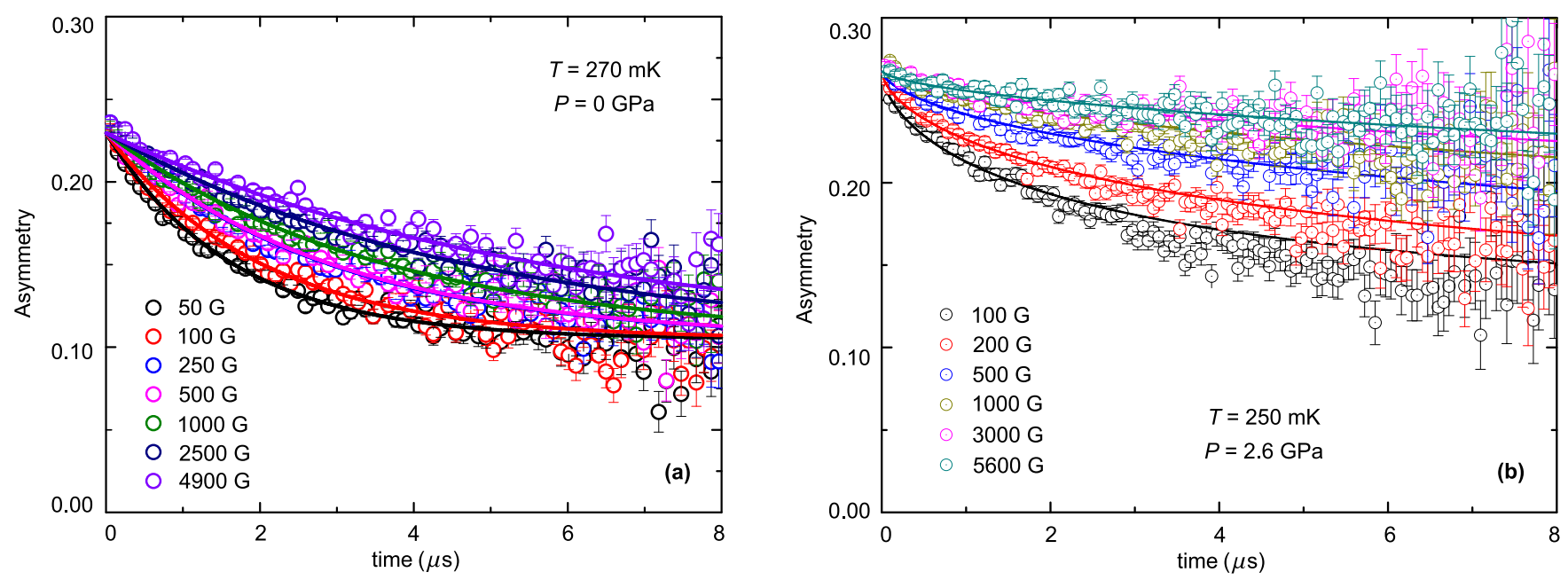

FIG. 6. Decoupling experiments (asymmetry as a function of time in different applied longitudinal fields) at (a) ambient pressure and $270 \mathrm{mK}$, and at (b) $2.6 \mathrm{GPa}$ and $250 \mathrm{mK}$. 
[1] L. Balents, Spin liquids in frustrated magnets, Nature (London) 464, 199 (2010).

[2] M. J. P. Gingras and P. A. McClarty, Quantum spin ice: A search for gapless quantum spin liquids in pyrochlore magnets, Rep. Prog. Phys. 77, 056501 (2014).

[3] L. Savary and L. Balents, Quantum spin liquids: A review, Rep. Prog. Phys. 80, 016502 (2017).

[4] M. Hermanns, I. Kimchi, and J. Knolle, Physics of the Kitaev model: Fractionalization, dynamic correlations, and material connections, Ann. Rev. Condens. Matter Phys. 9, 17 (2018).

[5] Y. Li, H. Liao, Z. Zhang, S. Li, F. Jin, L. Ling, L. Zhang, Y. Zou, L. Pi, Z. Yang, J. Wang, Z. Wu, and Q. Zhang, Gapless quantum spin liquid ground state in the two-dimensional spin$1 / 2$ triangular antiferromagnet $\mathrm{YbMgGaO}_{4}$, Sci. Rep. 5, 16419 (2015).

[6] Y. Li, G. Chen, W. Tong, L. Pi, J. Liu, Z. Yang, X. Wang, and Q. Zhang, Rare-Earth Triangular Lattice Spin Liquid: A SingleCrystal Study of $\mathrm{YbMgGaO}_{4}$, Phys. Rev. Lett. 115, 167203 (2015).

[7] Y. Li, P. Gegenwart, and A. A. Tsirlin, Spin liquids in geometrically perfect triangular antiferromagnets, J. Phys.: Condens. Matter 32, 224004 (2020).

[8] Y. Li, D. Adroja, P. K. Biswas, P. J. Baker, Q. Zhang, J. Liu, A. A. Tsirlin, P. Gegenwart, and Q. Zhang, Muon Spin Relaxation Evidence for the U(1) Quantum Spin-Liquid Ground State in the Triangular Antiferromagnet $\mathrm{YbMgGaO}_{4}$, Phys. Rev. Lett. 117, 097201 (2016)

[9] Z. Ma, J. Wang, Z.-Y. Dong, J. Zhang, S. Li, S.-H. Zheng, Y. Yu, W. Wang, L. Che, K. Ran, S. Bao, Z. Cai, P. Čermák, A. Schneidewind, S. Yano, J. S. Gardner, X. Lu, S.-L. Yu, J.-M. Liu, S. Li, J.-X. Li, and J. Wen, Spin-Glass Ground State in a Triangular-Lattice Compound $\mathrm{YbZnGaO}_{4}$, Phys. Rev. Lett. 120, 087201 (2018).

[10] Y. Li, S. Bachus, B. Liu, I. Radelytskyi, A. Bertin, A. Schneidewind, Y. Tokiwa, A. A. Tsirlin, and P. Gegenwart, Rearrangement of Uncorrelated Valence Bonds Evidenced by Low-Energy Spin Excitations in $\mathrm{YbMgGaO}_{4}$, Phys. Rev. Lett. 122, 137201 (2019).

[11] Y. Shen, Y.-D. Li, H. Wo, Y. Li, S. Shen, B. Pan, Q. Wang, H. C. Walker, P. Steffens, M. Boehm, Y. Hao, D. L. Quintero-Castro, L. W. Harriger, M. D. Frontzek, L. Hao, S. Meng, Q. Zhang, G. Chen, and J. Zhao, Evidence for a spinon Fermi surface in a triangular-lattice quantum-spin-liquid candidate, Nature (London) 540, 559 (2016).

[12] Y.-D. Li, Y.-M. Lu, and G. Chen, Spinon Fermi surface U(1) spin liquid in the spin-orbit-coupled triangular-lattice Mott insulator $\mathrm{YbMgGaO}_{4}$, Phys. Rev. B 96, 054445 (2017).

[13] Y.-D. Li and G. Chen, Detecting spin fractionalization in a spinon Fermi surface spin liquid, Phys. Rev. B 96, 075105 (2017).

[14] Y. Shen, Y.-D. Li, H. C. Walker, P. Steffens, M. Boehm, X. Zhang, S. Shen, H. Wo, G. Chen, and J. Zhao, Fractionalized excitations in the partially magnetized spin liquid candidate $\mathrm{YbMgGaO}_{4}$, Nat. Commun. 9, 4138 (2018).

[15] Y. Li, D. Adroja, D. Voneshen, R. I. Bewley, Q. Zhang, A. A. Tsirlin, and P. Gegenwart, Nearest-neighbour resonating valence bonds in $\mathrm{YbMgGaO}_{4}$, Nat. Commun. 8, 15814 (2017).

[16] P. W. Anderson, Resonating valence bonds: A new kind of insulator? Mater. Res. Bull. 8, 153 (1973).
[17] L. Ding, P. Manuel, S. Bachus, F. Grußler, P. Gegenwart, J. Singleton, R. D. Johnson, H. C. Walker, D. T. Adroja, A. D. Hillier, and A. A. Tsirlin, Gapless spin-liquid state in the structurally disorder-free triangular antiferromagnet $\mathrm{NaYbO}_{2}$, Phys. Rev. B 100, 144432 (2019).

[18] J. Ma, J. Li, Y. H. Gao, C. Liu, Q. Ren, Z. Zhang, Z. Wang, R. Chen, J. Embs, E. Feng, F. Zhu, Q. Huang, Z. Xiang, L. Chen, E. S. Choi, Z. Qu, L. Li, J. Wang, H. Zhou, Y. Su, X. Wang, Q. Zhang, and G. Chen, Spin-orbit-coupled triangular-lattice spin liquid in rare-earth chalcogenides, arXiv:2002.09224.

[19] P.-L. Dai, G. Zhang, Y. Xie, C. Duan, Y. Gao, Z. Zhu, E. Feng, C.-L. Huang, H. Cao, A. Podlesnyak, G. E. Granroth, D. Voneshen, S. Wang, G. Tan, E. Morosan, X. Wang, L. Shu, G. Chen, Y. Guo, X. Lu, and P. Dai, Spinon Fermi surface spin liquid in a triangular lattice antiferromagnet $\mathrm{NaYbSe}_{2}$, arXiv:2004.06867.

[20] Y. Li, D. Adroja, R. I. Bewley, D. Voneshen, A. A. Tsirlin, P. Gegenwart, and Q. Zhang, Crystalline Electric-Field Randomness in the Triangular Lattice Spin-Liquid $\mathrm{YbMgGaO}_{4}$, Phys. Rev. Lett. 118, 107202 (2017).

[21] J. A. M. Paddison, M. Daum, Z. Dun, G. Ehlers, Y. Liu, M. B. Stone, H. Zhou, and M. Mourigal, Continuous excitations of the triangular-lattice quantum spin liquid $\mathrm{YbMgGaO}_{4}$, Nat. Phys. 113, 117 (2017).

[22] See Supplemental Material at http://link.aps.org/supplemental/ 10.1103/PhysRevResearch.2.023191 for the crystallographic cif file with crystal structures refined at different pressures.

[23] R. J. Angel, J. Gonzalez-Platas, and M. Alvaro, EosFit7c and a Fortran module (library) for equation of state calculations, $\mathrm{Z}$. Krist. 229, 405 (2014).

[24] A. K. Mishra, H. K. Poswal, S. M. Sharma, S. Saha, D. V. S. Muthu, S. Singh, R. Suryanarayanan, A. Revcolevschi, and A. K. Sood, The study of pressure induced structural phase transition in spin-frustrated $\mathrm{Yb}_{2} \mathrm{Ti}_{2} \mathrm{O}_{7}$ pyrochlore, J. Appl. Phys. 111, 033509 (2012).

[25] K. M. Turner, D. R. Rittman, R. A. Heymach, C. L. Tracy, M. L. Turner, A. F. Fuentes, W. L. Mao, and R. C. Ewing, Pressure-induced structural modifications of rare-earth hafnate pyrochlore, J. Phys.: Condens. Matter 29, 255401 (2017).

[26] R. Khasanov, Z. Guguchia, A. Maisuradze, D. Andreica, M. Elender, A. Raselli, Z. Shermadini, T. Goko, F. Knecht, E. Morenzoni, and A. Amato, High pressure research using muons at the Paul Scherrer Institute, High Press. Res. 36, 140 (2016).

[27] R. Orbach, Spin-lattice relaxation in rare-earth salts, Proc. R. Soc. London, Ser. A 264, 458 (1961).

[28] A. Keren, P. Mendels, I. A. Campbell, and J. Lord, Probing the Spin-Spin Dynamical Autocorrelation Function in a Spin Glass above $T_{g}$ via Muon Spin Relaxation, Phys. Rev. Lett. 77, 1386 (1996).

[29] The data above $t=6 \mu$ s were excluded due to their large error bars.

[30] A. Keren, Muons as probes of dynamical spin fluctuations: Some new aspects, J. Phys.: Condens. Matter 16, S4603 (2004).

[31] D. E. MacLaughlin, R. H. Heffner, O. O. Bernal, K. Ishida, J. E. Sonier, G. J. Nieuwenhuys, M. B. Maple, and G. R. Stewart, Disorder, inhomogeneity and spin dynamics in $f$-electron nonFermi liquid systems, J. Phys.: Condens. Matter 16, S4479 (2004).

[32] P. Mendels and F. Bert, Quantum kagome antiferromagnet $\mathrm{ZnCu}_{3}(\mathrm{OH})_{6} \mathrm{Cl}_{2}$, J. Phys. Soc. Jpn. 79, 011001 (2010). 
[33] M. R. Norman, Colloquium: Herbertsmithite and the search for the quantum spin liquid, Rev. Mod. Phys. 88, 041002 (2016).

[34] D. P. Kozlenko, A. F. Kusmartseva, E. V. Lukin, D. A. Keen, W. G. Marshall, M. A. de Vries, and K. V. Kamenev, From Quantum Disorder to Magnetic Order in an $S=\frac{1}{2}$ Kagome Lattice: A Structural and Magnetic Study of Herbertsmithite at High Pressure, Phys. Rev. Lett. 108, 187207 (2012).

[35] J. G. Rau and M. J. P. Gingras, Frustrated quantum rare-earth pyrochlores, Ann. Rev. Condens. Matter Phys. 10, 357 (2019).

[36] E. Kermarrec, J. Gaudet, K. Fritsch, R. Khasanov, Z. Guguchia, C. Ritter, K. A. Ross, H. A. Dabkowska, and B. D. Gaulin, Ground state selection under pressure in the quantum pyrochlore magnet $\mathrm{Yb}_{2} \mathrm{Ti}_{2} \mathrm{O}_{7}$, Nat. Commun. 8, 14810 (2017).

[37] Q. Luo, S. Hu, B. Xi, J. Zhao, and X. Wang, Ground-state phase diagram of an anisotropic spin- $\frac{1}{2}$ model on the triangular lattice, Phys. Rev. B 95, 165110 (2017).

[38] Z. Zhu, P. A. Maksimov, S. R. White, and A. L. Chernyshev, Topography of Spin Liquids on a Triangular Lattice, Phys. Rev. Lett. 120, 207203 (2018).

[39] P. A. Maksimov, Z. Zhu, S. R. White, and A. L. Chernyshev, Anisotropic-Exchange Magnets on a Triangular Lattice: Spin Waves, Accidental Degeneracies, and Dual Spin Liquids, Phys. Rev. X 9, 021017 (2019).

[40] Z. Zhu, P. A. Maksimov, S. R. White, and A. L. Chernyshev, Disorder-Induced Mimicry of a Spin Liquid in $\mathrm{YbMgGaO}_{4}$, Phys. Rev. Lett. 119, 157201 (2017).

[41] X. Zhang, F. Mahmood, M. Daum, Z. Dun, J. A. M. Paddison, N. J. Laurita, T. Hong, H. Zhou, N. P. Armitage, and M. Mourigal, Hierarchy of Exchange Interactions in the Triangular-Lattice Spin Liquid $\mathrm{YbMgGaO}_{4}$, Phys. Rev. X 8, 031001 (2018).

[42] J. G. Rau and M. J. P. Gingras, Frustration and anisotropic exchange in ytterbium magnets with edge-shared octahedra, Phys. Rev. B 98, 054408 (2018).

[43] M. E. Zayed, C. Rüegg, J. Larrea J., A. M. Läuchli, C. Panagopoulos, S. S. Saxena, M. Ellerby, D. F. McMorrow, T. Strässle, S. Klotz, G. Hamel, R. A. Sadykov, V.
Pomjakushin, M. Boehm, M. Jiménez-Ruiz, A. Schneidewind, E. Pomjakushina, M. Stingaciu, K. Conder, and H. M. Rønnow, 4-spin plaquette singlet state in the Shastry-Sutherland compound $\mathrm{SrCu}_{2}\left(\mathrm{BO}_{3}\right)_{2}$, Nat. Phys. 13, 962 (2017).

[44] S. A. Zvyagin, D. Graf, T. Sakurai, S. Kimura, H. Nojiri, J. Wosnitza, H. Ohta, T. Ono, and H. Tanaka, Pressure-tuning the quantum spin Hamiltonian of the triangular lattice antiferromagnet $\mathrm{Cs}_{2} \mathrm{CuCl}_{4}$, Nat. Commun. 10, 1064 (2019).

[45] D. A. Prishchenko, A. A. Tsirlin, V. Tsurkan, A. Loidl, A. Jesche, and V. G. Mazurenko, Antiferroelectric instability in the kagome francisites $\mathrm{Cu}_{3} \mathrm{Bi}\left(\mathrm{SeO}_{3}\right)_{2} \mathrm{O}_{2} X(X=\mathrm{Cl}, \mathrm{Br})$, Phys. Rev. B 95, 064102 (2017).

[46] Rigaku Oxford Diffraction, CrysAlisPro Software system, version 1.171.37.35 (Rigaku Corporation, Oxford, UK, 2014).

[47] G. M. Sheldrick, A history of SHELX, Acta Cryst. A 64, 112 (2008).

[48] C. B. Hübschle, G. M. Sheldrick, and B. Dittrich, ShelXle: A Qt graphical user interface for SHELXL, J. Appl. Cryst. 44, 1281 (2011).

[49] K. Koepernik and H. Eschrig, Full-potential nonorthogonal local-orbital minimum-basis band-structure scheme, Phys. Rev. B 59, 1743 (1999).

[50] G. Kresse and J. Furthmüller, Efficiency of ab initio total energy calculations for metals and semiconductors using a plane-wave basis set, Comput. Mater. Sci. 6, 15 (1996); Efficient iterative schemes for $a b$ initio total-energy calculations using a planewave basis set, Phys. Rev. B 54, 11169 (1996).

[51] J. P. Perdew and Y. Wang, Accurate and simple analytic representation of the electron-gas correlation energy, Phys. Rev. B 45, 13244 (1992).

[52] J. P. Perdew, K. Burke, and M. Ernzerhof, Generalized Gradient Approximation Made Simple, Phys. Rev. Lett. 77, 3865 (1996).

[53] Z. Shermadini, R. Khasanov, M. Elender, G. Simutis, Z. Guguchia, K. V. Kamenev, and A. Amato, A low-background piston-cylinder type hybrid high pressure cell for muon-spin rotation/relaxation experiments, High Press. Res. 37, 449 (2017). 